Weili Li* and Yang Bo

\title{
Crystal structure of $\left\{2,2^{\prime}-\{c y c l o h e x a n e-1,2\right.$-diylbis [(azanylylidene)methylylidene]\}bis(2,4- dibromophenolato)- $\mathrm{K}^{4} \mathrm{~N}, \mathrm{~N}^{\prime}, 0, \mathrm{O}^{\prime}$ \} copper(II) - diethylformamide (1/1), $\mathrm{C}_{23} \mathrm{H}_{23} \mathrm{Br}_{4} \mathrm{CuN}_{3} \mathrm{O}_{3}$
}

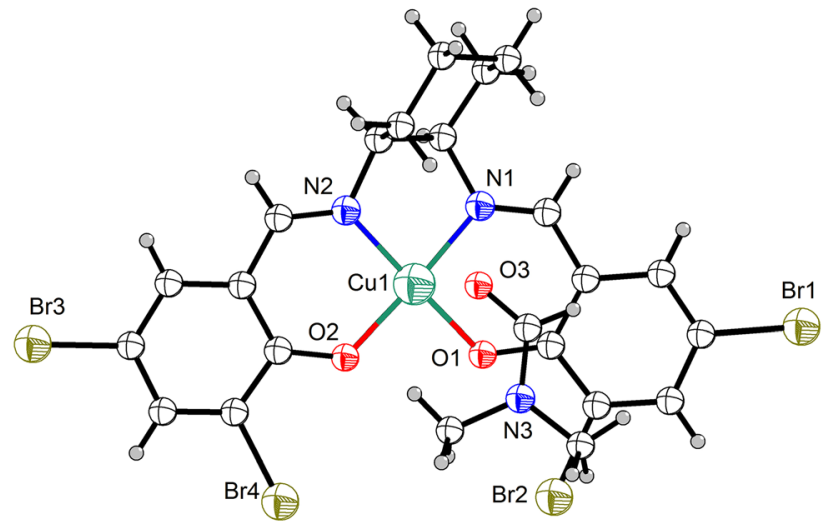

https://doi.org/10.1515/ncrs-2021-0379

Received September 29, 2021; accepted November 21, 2021;

published online February 14, 2022

\section{Abstract}

$\mathrm{C}_{23} \mathrm{H}_{23} \mathrm{Br}_{4} \mathrm{CuN}_{3} \mathrm{O}_{3}, \quad P \overline{1} \quad$ (no. 2), $a=10.4769(4) \AA$, $b=10.7829(5) \AA, c=12.9824(5) \AA, \alpha=98.153(2)^{\circ}$, $\beta=111.484(2)^{\circ}, \gamma=105.035(2)^{\circ}, V=1271.43(9) \AA^{3}, Z=2$, $R_{g t}(F)=0.0338, \mathrm{w} R_{\text {ref }}\left(F^{2}\right)=0.1158, T=273.15 \mathrm{~K}$.

CCDC no.: 2111978

The molecular structure is shown in the figure. Table 1 contains crystallographic data and Table 2 contains the list of the atoms including atomic coordinates and displacement parameters.

\section{Source of material}

The structure was solved and refined with the OLEX2 program [2] as an interface together with the SHELXT and SHELXL

\footnotetext{
*Corresponding author: Weili Li, Department of Chemical Science and Technology, Kunming University, Yunnan, Kunming 65200, P. R. China, E-mail: Iwlkmu@163.com. https://orcid.org/0000-0003 0700-3127

Yang Bo, Department of Chemical Science and Technology, Kunming University, Yunnan, Kunming 65200, P. R. China
}

Table 1: Data collection and handling.

\begin{tabular}{ll}
\hline Crystal: & Block, clear blackish black \\
Size: & $0.16 \times 0.14 \times 0.12 \mathrm{~mm}$ \\
Wavelength: & MoK $\alpha$ radiation $(0.71073 \AA)$ \\
$\mu:$ & $7.18 \mathrm{~mm}^{-1}$ \\
Diffractometer, scan mode: & Agilent CCD detector, $\varphi$ and $\omega$-scans \\
$\theta_{\text {max }}$, completeness: & $26.4^{\circ},>99 \%$ \\
$N\left(h k l_{\text {measured }}, N\left(h k l_{\text {unique }}, R_{\text {int }}:\right.\right.$ & $8469,5230,0.022$ \\
Criterion for $I_{\text {obs }}, N(h k l)_{\text {gt }}:$ & $I_{\text {obs }}>2 \sigma\left(I_{\text {obs }}\right), 4520$ \\
$N(\text { param })_{\text {refined }}:$ & 309 \\
Programs: & CrysAlis ${ }^{\mathrm{PRO}}[1]$, OLEX2 [2], SHELX [3, 4] \\
\hline
\end{tabular}

programs $[3,4]$. All $\mathrm{H}$ atoms were placed in geometrically idealized positions and refined using a riding model.

\section{Experimental details}

3,5-Dibromosalicylaldehyde (0.0263 g, $0.094 \mathrm{mmol})$ was dissolved in methanol $(30 \mathrm{~mL})$ with stirring, adding 1,2-cyclohexanediamine $(0.047 \mathrm{mmol})$. The mixture was stirred and refluxed at $80{ }^{\circ} \mathrm{C}$ for $3 \mathrm{~h}$. The solvent was removed using a rotary evaporator to give the Schiff-base ligand (+-)-3,5-BrsalcyH $\mathrm{H}_{2}$. After this(+-)-3,5-BrsalcyH $\mathrm{H}_{2}$ $(0.1 \mathrm{mmol})$ was dissolved in a mixture containing $20 \mathrm{~mL}$ $\mathrm{N}, \mathrm{N}$-dimethylformamide (DMF) and $10 \mathrm{~mL}$ ethanol. Then, $0.1 \mathrm{~g}$ copper nitrate was add to the above solution and the resulting dark green mixture was further stirred at room temperature overnight. Dark brown crystals of the title compound were isolated after two weeks.

\section{Comment}

Schiff bases have a variety of chemical and biologica activities [5, 6]. The research concerning halogenated salentype $\mathrm{Cu}$-complexes is still at an early stage [7-10]. As a part of our current research interest on the regulating effect of Schiff base ligands on transition metal complexes, we report herein a new $\mathrm{Cu}(\mathrm{II})$ complex. 
Table 2: Fractional atomic coordinates and isotropic or equivalent isotropic displacement parameters $\left(\AA^{2}\right)$.

\begin{tabular}{|c|c|c|c|c|}
\hline Atom & $x$ & $y$ & $z$ & $U_{\text {iso }} * / U_{\text {eq }}$ \\
\hline $\mathrm{Br} 2$ & $0.63313(5)$ & $0.23839(5)$ & $0.73782(4)$ & $0.01910(13)$ \\
\hline Br1 & $0.96491(6)$ & $0.70727(5)$ & $1.09894(4)$ & 0.02541 (14) \\
\hline $\mathrm{Br} 4$ & $0.18198(6)$ & $0.01430(5)$ & $0.34252(5)$ & $0.02474(14)$ \\
\hline Cu1 & $0.34896(6)$ & $0.48150(6)$ & $0.52712(5)$ & $0.01562(15)$ \\
\hline $\mathrm{Br} 3$ & $-0.28775(7)$ & $0.07518(6)$ & $-0.01305(5)$ & 0.04215 (19) \\
\hline 02 & $0.2553(4)$ & 0.3058 & $0.4296(3)$ & $0.0197(7)$ \\
\hline 01 & $0.4954(4)$ & $0.4229(3)$ & $0.6236(3)$ & $0.0187(7)$ \\
\hline N1 & $0.4484(4)$ & $0.6665(4)$ & $0.6160(3)$ & $0.0163(8)$ \\
\hline N2 & $0.2062(5)$ & $0.5473(4)$ & $0.4296(4)$ & 0.0178 (9) \\
\hline $\mathrm{C} 3$ & $0.7808(6)$ & $0.4841(5)$ & $0.9077(4)$ & $0.0189(10)$ \\
\hline H3 & 0.8319 & 0.4369 & 0.9510 & $0.023^{*}$ \\
\hline C13 & $0.2303(5)$ & $0.6883(4)$ & $0.4780(4)$ & $0.0143(9)$ \\
\hline H13 & 0.1973 & 0.7297 & 0.4152 & $0.017^{*}$ \\
\hline C9 & $0.4276(6)$ & $0.8976(5)$ & $0.6178(5)$ & $0.0212(11)$ \\
\hline H9A & 0.4057 & 0.9526 & 0.5652 & $0.025^{\star}$ \\
\hline H9B & 0.5307 & 0.9354 & 0.6685 & $0.025^{*}$ \\
\hline C4 & $0.8110(5)$ & $0.6180(5)$ & $0.9512(4)$ & $0.0180(10)$ \\
\hline $\mathrm{C} 1$ & $0.5909(6)$ & $0.4879(5)$ & $0.7264(4)$ & $0.0175(10)$ \\
\hline N3 & $0.2973(6)$ & $0.3072(5)$ & $0.7716(5)$ & $0.0318(11)$ \\
\hline $\mathrm{C} 20$ & $0.1406(5)$ & $0.2638(5)$ & $.3338(4)$ & $0.0161(9)$ \\
\hline C7 & $0.5559(6)$ & $0.7073(5)$ & $0.7140(4)$ & $0.0185(10)$ \\
\hline H7 & 0.5935 & 0.7983 & 0.7484 & $0.022^{\star}$ \\
\hline $\mathrm{C} 8$ & $0.3952(5)$ & $0.7578(5)$ & $0.5499(4)$ & $0.0153(9)$ \\
\hline H8 & 0.4406 & 0.7662 & 0.4961 & $0.018^{*}$ \\
\hline C19 & $0.0851(5)$ & $0.1270(5)$ & $0.2726(4)$ & $0.0154(9)$ \\
\hline C2 & $0.6729(5)$ & $0.4218(5)$ & $0.7982(4)$ & $0.0165(10)$ \\
\hline C15 & $0.0592(5)$ & $0.3421(5)$ & $0.2807(4)$ & $0.0155(9)$ \\
\hline C6 & $0.6252(5)$ & $0.6240(4)$ & $0.7768(4)$ & $0.0139(9)$ \\
\hline C14 & $0.0969(5)$ & $0.4817(5)$ & $0.3332(4)$ & $0.0167(10)$ \\
\hline H14 & 0.0384 & 0.5271 & 0.2949 & $0.020^{*}$ \\
\hline C18 & $-0.0358(6)$ & 0.0709 (5) & $0.1701(4)$ & $0.0196(10)$ \\
\hline H18 & -0.0662 & -0.0188 & 0.1330 & $0.023^{*}$ \\
\hline 03 & $0.2322(6)$ & $0.4707(5)$ & $0.6960(6)$ & 0.0577 (15) \\
\hline C10 & $0.3399(6)$ & $0.9002(5)$ & $0.6886(5)$ & $0.0223(11)$ \\
\hline $\mathrm{H} 10 \mathrm{~A}$ & 0.3660 & 0.8503 & 0.7449 & $0.027^{\star}$ \\
\hline $\mathrm{H} 10 \mathrm{~B}$ & 0.3625 & 0.9914 & 0.7293 & $0.027^{\star}$ \\
\hline $\mathrm{C} 5$ & $0.7374(5)$ & $0.6894(5)$ & $0.8889(4)$ & $0.0178(10)$ \\
\hline H5 & 0.7606 & 0.7799 & 0.9194 & $0.021^{*}$ \\
\hline C17 & $-0.1118(6)$ & $0.1533(5)$ & $0.1234(4)$ & $0.0244(11)$ \\
\hline C12 & $0.1428(6)$ & $0.6958(5)$ & $0.5493(5)$ & $0.0233(11)$ \\
\hline $\mathrm{H} 12 \mathrm{~A}$ & 0.0394 & 0.6575 & 0.4994 & $0.028^{*}$ \\
\hline $\mathrm{H} 12 \mathrm{~B}$ & 0.1655 & 0.6445 & 0.6050 & $0.028^{*}$ \\
\hline C11 & $0.1789(6)$ & $0.8403(5)$ & $0.6115(5)$ & $0.0221(11)$ \\
\hline $\mathrm{H} 11 \mathrm{~A}$ & 0.1524 & 0.8910 & 0.5558 & $0.026^{*}$ \\
\hline $\mathrm{H} 11 \mathrm{~B}$ & 0.1235 & 0.8438 & 0.6568 & $0.026^{*}$ \\
\hline C16 & $-0.0666(6)$ & $0.2866(6)$ & $0.1770(4)$ & $0.0226(11)$ \\
\hline H16 & -0.1192 & 0.3394 & 0.1447 & $0.027^{\star}$ \\
\hline C24 & $0.3728(8)$ & $0.2668(7)$ & $0.8723(6)$ & 0.0367 (15) \\
\hline $\mathrm{H} 24 \mathrm{~A}$ & 0.3045 & 0.1961 & 0.8821 & $0.055^{\star}$ \\
\hline H24B & 0.4166 & 0.3412 & 0.9388 & $0.055^{\star}$ \\
\hline $\mathrm{H} 24 \mathrm{C}$ & 0.4471 & 0.2365 & 0.8626 & $0.055^{\star}$ \\
\hline C23 & $0.2168(7)$ & $0.2056(7)$ & $0.6616(6)$ & $0.0412(16)$ \\
\hline $\mathrm{H} 23 \mathrm{~A}$ & 0.2843 & 0.1784 & 0.6379 & $0.062^{\star}$ \\
\hline H23B & 0.1608 & 0.2417 & 0.6049 & $0.062^{\star}$ \\
\hline $\mathrm{H} 23 \mathrm{C}$ & 0.1526 & 0.1301 & 0.6701 & $0.062^{\star}$ \\
\hline
\end{tabular}

Table 2: (continued)

\begin{tabular}{lrrrr}
\hline Atom & $\boldsymbol{x}$ & $\boldsymbol{y}$ & $\boldsymbol{z}$ & $\boldsymbol{U}_{\text {iso }} \boldsymbol{U}_{\text {eq }}$ \\
\hline $\mathrm{C} 21$ & $0.2945(8)$ & $0.4308(7)$ & $0.7783(7)$ & $0.0433(17)$ \\
$\mathrm{H} 21$ & 0.3429 & 0.4913 & 0.8503 & $0.052^{*}$ \\
\hline
\end{tabular}

The basic molecular structure of the title compound shows a classic $\mathrm{Cu}$-salen complex configuration, in which the copper center possesses tetra-coordinated environment. Neighbouring monomers are further linked by weak intermolecular halogen-bonding interactions: $\mathrm{Br} 1 \cdots \mathrm{Br} 4^{\mathrm{i}}, \mathrm{D} \cdots \mathrm{A}$ : 3.740(2) $\AA$; Br2 ․ Br $3^{\text {ii }}, \mathrm{D} \cdots \mathrm{A}: 3.740(2) \AA$, symmetry code: (i): symmetry code: (i): $(1+x, 1+y, 1+z)$. Another interesting feature of the title compound is the DMF solvent molecules located between $\mathrm{Cu}$-salen molecules contributing the stability of the supramolecular networks.

According to the result of PLATON software [19], the classic $\pi-\pi$ stacking interactions is another important driving force in linking the adjacency molecules in to supermolecular networks.

Author contributions: All the authors have accepted responsibility for the entire content of this submitted manuscript and approved submission.

Research funding: We gratefully acknowledge the financial support from the Joint Basic Research Program (partial) of Yunnan Provincial Undergraduate Universities (202101BA070001-052, 202001A0070059), and the Program for Innovative Research Team (in Science and Technology) in Universities of Yunnan Province.

Conflict of interest statement: The authors declare no conflicts of interest regarding this article.

\section{References}

1. Agilent Technologies. CrysAlis ${ }^{P R O}$ Software System; Agilent Technologies UK Ltd: Oxford, UK, 2015.

2. Dolomanov O. V., Bourhis L. J., Gildea R. J., Howard J. A. K., Puschmann H. OLEX2: a complete structure solution, refinement and analysis program. J. Appl. Crystallogr. 2010, 42, 339-341.

3. Sheldrick G. A short history of SHELX. Acta Crystallogr. 2008, A64, 112-122.

4. Sheldrick G. Crystal structure refinement with SHELXL. Acta Crystallogr. C 2015, 71, 3-8.

5. Miyasaka H., Saitoh A., Abe S. Magnetic assemblies based on Mn(III) salen analogues. Coord. Chem. Rev. 2007, 251, 2622-2664.

6. Nemec V., Fotovi L., Friščić T., Cinčić D. A large family of halogenbonded cocrystals involving metal-organic building blocks with open coordination sites. Cryst. Growth Des. 2017, 17, 6169-6173. 
7. Wu Q., Li J., Liu F., Xiao J., Tang Y., Zi Q. Synthesis, crystallographic structure, Hirshfeld surface analysis, and DFT calculations of two Salen-type hetero-halogenated Schiff base Mn(IV)/(III) complexes. Russ. J. Coord. Chem. 2020, 46, 137-144.

8. Wu Q., Tang Y., Zi Q. Synthesis, crystallographic structure, Hirshfeld surface analysis and DFT calculations of two Salen-type halogenated Schiff-base Ni(II) complexes. Polyhedron 2019, 166, 123-129.
9. Tohidiyan Z., Sheikhshoaie I., Khaleghi M., Mague J. T. A novel copper (II) complex containing a tetradentate Schiff base: synthesis, spectroscopy, crystal structure, DFT study, biological activity and preparation of its nano-sized metal oxide. J. Mol. Struct. 2017, 1134, 706-714.

10. Mandal S., Layek M., Saha R., Rizzoli C., Bandyopadhyay D. Synthesis, crystal structure and antibacterial activity of four mononuclear Schiff base complexes of copper(II) and nickel(II). Transition Met. Chem. 2021, 46, 9-16. 